\title{
ANALYTICAL EVALUATION OF ECONOMIC RISK CAPITAL FOR PORTFOLIOS OF GAMMA RISKS
}

\author{
BY \\ WERNER HÜRLIMANN
}

\begin{abstract}
Based on the notions of value-at-risk and expected shortfall, we consider two functionals, abbreviated $\mathrm{VaR}$ and $\mathrm{RaC}$, which represent the economic risk capital of a risky business over some time period required to cover losses with a high probability. These functionals are consistent with the risk preferences of profit-seeking (and risk averse) decision makers and preserve the stochastic dominance order (and the stop-loss order). Quantitatively, $\mathrm{RaC}$ is equal to $\mathrm{VaR}$ plus an additional stop-loss dependent term, which takes into account the average amount at loss. Furthermore, $\mathrm{RaC}$ is additive for comonotonic risks, which is an important extremal situation encountered in the modeling of dependencies in multivariate risk portfolios. Numerical illustrations for portfolios of gamma distributed risks follow. As a result of independent interest, new analytical expressions for the exact probability density of sums of independent gamma random variables are included, which are similar but different to previous expressions by Provost (1989) and Sim (1992).
\end{abstract}

\section{KEYWORDS}

Value-at-risk, expected shortfall, risk-adjusted capital, comonotonicity, additivity, supermodular order, stop-loss order, gamma convolutions.

\section{ECONOMIC RISK CAPITAL USING VAR AND RAC}

Suppose a firm is confronted with a risky business over some time period, and let the random variable $X$ represent the potential loss or risk the firm incurs at the end of the period. To be able to cover any loss with a high probability, the firm borrows at the beginning of the time period on the capital market the amount $E R C_{0}$, called economic risk capital. At the end of the period, the firm has to pay interest on this at the interest rate $i_{R}$. To guarantee with certainty the value of the borrowed capital at the end of the period, the firm invests $E R C_{0}$ at the risk-free interest rate $i_{f}<i_{R}$. The value of the economic risk capital at the end of the period is thus $E R C=E R C_{0} \cdot\left(1+i_{f}-i_{R}\right)$. The risky business will 
be successful at the end of the period provided the event $\{X>E R C\}$ occurs only with a small tolerance probability.

There exist several risk management principles applied to evaluate $E R C$. Two simple methods that have been considered so far are the value-at-risk and the expected shortfall approach (e.g. Arztner et al. (1997a/b), Arztner (1999), Embrechts (1995), Hürlimann (1998a), Schröder (1996), Wirch (1999)). According to the value-at-risk method one identifies the economic risk capital with the value-at-risk of the loss setting

$$
E R C=V A R_{a}[X]:=Q_{X}(\alpha),
$$

where $Q_{X}(u)=\inf \left\{x \mid F_{X}(x) \geq u\right\}$ is a quantile function of $X$, with $F_{X}(x)=$ $\operatorname{Pr}(X \leq x)$ the distribution of $X$. This quantile represents the maximum possible loss, which is not exceeded with the (high) probability a (called security level). According to the expected shortfall method one identifies the economic risk capital with the risk-adjusted capital of the loss setting

$$
E R C=\operatorname{RaC}_{a}[X]:=E\left[X \mid X>\operatorname{VaR}_{a}[X]\right] .
$$

This value represents the conditional expected loss given the loss exceeds its value-at-risk. Clearly one has

$$
R a C_{a}[X]=Q_{X}(\alpha)+m_{X}\left[Q_{X}(\alpha)\right]=Q_{X}(\alpha)+\frac{1}{\varepsilon} \pi_{X}\left[Q_{X}(\alpha)\right],
$$

where $m_{X}(x)=E[X-x \mid X>x]$ is the mean excess function, $\pi_{X}(x)=\left(1-F_{X}(x)\right)$. $m_{X}(x)$ is the stop-loss transform, and $\varepsilon=1-\alpha$ is interpreted as loss probability (called loss tolerance level). In Arztner (1999) the expression (4.3) is called tail conditional expectation and abbreviated TailVaR there (for tail value-at-risk). Mathematically, $\mathrm{VaR}$ and $\mathrm{RaC}$, which have been defined as functions of random variables, may be viewed as functionals defined on the space of probability distributions associated with these random variables. By abuse of language, we will use the terminology functionals when appropriate.

It is important to observe that both $E R C$ functionals satisfy two important risk-preference criteria in the economics of insurance (see Denuit et al. (1999) for a recent review). They are consistent with the risk preferences of profit-seeking decision makers respectively profit-seeking risk averse decision makers. To see this let us first recall two partial orders of riskiness.

Definitions 1.1. A risk $X$ is less dangerous than a risk $Y$ in the stochastic order, written $X \leq_{s t} Y$, if $Q_{X}(u) \leq Q_{Y}(u)$ for all $u \in[0,1]$. A risk $X$ is less dangerous than a risk $Y$ in the stop-loss order, written $X \leq s l Y$, if $\pi_{X}(x) \leq \pi_{Y}(x)$ for all $x$.

To compare economic risk capitals using criteria, which do not depend on the choice of the loss tolerance level, let us introduce two further partial orders of riskiness.

Definitions 1.2. A loss $X$ is less dangerous than a loss $Y$ in the VaR order, written $X \leq_{V a R} Y$, if the value-at-risk quantities satisfy $\operatorname{VaR}_{a}[X] \leq \operatorname{VaR}_{u}[Y]$, for 
all $a \in[0,1]$. A loss $X$ is less dangerous than a loss $Y$ in the $R a C$ order, written $X \leq_{R a C} Y$, if the risk-adjusted capital quantities satisfy $R a C_{a}[X] \leq R a C_{a}[Y]$, for all $a \in[0,1]$.

The value-at-risk and expected shortfall methods are consistent with ordering of risks in the sense that profit-seeking (risk averse) decision makers require higher VaR $(\mathrm{RaC})$ by increasing risk, where risk is compared using the stochastic order $\leq_{s t}$ (stop-loss order $\leq_{s t}$ ). Reciprocally, increasing VaR $(\mathrm{RaC})$ is always coupled with higher risk. The following result expresses these ordering properties mathematically.

Theorem 1.1. If $X$ and $Y$ are two loss random variables, then $X \leq_{V a R} Y \Leftrightarrow X \leq_{s t} Y$ and $X \leq_{R a C} Y \Leftrightarrow X \leq_{s l} Y$.

Proof. Since $X \leq_{s i} Y \Leftrightarrow Q_{X}(u) \leq Q_{Y}(u)$ for all $a \in[0,1]$, the first property is immediate by (1.1). Consider the Hardy-Littlewood transform defined by

$$
H L_{X}(u)=\left\{\begin{array}{l}
\frac{1}{1-u} \cdot \int_{u}^{1} Q_{X}(t) d t, u<1 \\
Q_{X}(1), u=1
\end{array}\right.
$$

Its name stems from the Hardy-Littlewood (1930) maximal function and has been extensively used in both theoretical and applied mathematics (e.g. Blackwell and Dubins (1963), Dubins and Gilat (1978), Meilijson and Nàdas (1979), Kertz and Rösler (1990/92/93), Rüschendorf (1991), Hürlimann (1998b/c/d)). One knows that there exists a random variable $X^{H}$ associated to $X$ such that (e.g. Hürlimann (1998b), Theorem 2.1)

$$
H L_{X}(u)=Q_{X^{H}}(u)=Q_{X}(u)+m_{X}\left[Q_{X}(u],\right.
$$

hence $R A C_{a}[X]=Q_{X^{H}}(a)$ by (1.3). The result follows from the fact that $X \leq_{s l} Y$ if and only if $X^{H} \leq_{s t} Y^{H}$, where $\leq_{s t}$ denotes the usual stochastic dominance order (e.g Kertz and Rösler (1992), Lemma 1.8, or Hürlimann (1998c), Theorem 2.3). For the convenience of the reader, an alternative perhaps more accessible proof should also be pointed out. Consider the so-called distortion function $g_{a}(x)=\min \left\{\frac{x}{1-\alpha}, 1\right\}$. It is easy to show that

$$
\operatorname{RaC}_{a}[X]=\int_{0}^{\infty} g_{a}\left[1-F_{X}(x)\right] d x
$$

identifies the $\mathrm{RaC}$ functional with a member of the class of distortion pricing principles in Wang (1996). The result follows by Dhaene et al. (2000), Theorem 3 , which contains a proof of the stated equivalence. $\diamond$

Finally, it is important to observe that, except for a world of elliptical linear portfolio losses (Embrechts et al. (1998), Fundamental Theorem of Risk Management), the VaR functional has several shortcomings. It is not subadditive 
and not scalar multiplicative, and it cannot discriminate between risk-averse and risk-taking portfolios (examples 1 to 3 in Wirch (1999)). Some more details for the practitioner are in order. Recall that a risk measure $R[\cdot]$ acting on the set of all risks is subadditive provided $R[X+Y] \leq R[X]+R[Y]$ for all $X, Y$, that is merging two risks does not create extra risk. If a firm must meet a requirement of extra economic risk capital that did not satisfy this property, the firm might separate in two subunits requiring less capital, a matter of concern for the supervising authority. A risk measure is scalar multiplicative provided $R[c X]=c R[X]$ for all $X$, all constants $c \geq 0$. In situations where no diversification occurs capital requirement depends on the size of the risk. In contrast to this, the $\mathrm{RaC}$ functional, which is subadditve and scalar multiplicative, is a coherent risk measure in the sense of Arztner et al. (1997) and appears thus more suitable in general applications. A recent work devoted to the evaluation of economic risk capital in life-insurance using the $\mathrm{VaR}$ and $\mathrm{RaC}$ approaches is Ballmann and Hürlimann (2000).

\section{The MAXimum RaC FOR THE AGgREGate RISK OF PORTFOlios}

An important but complex problem is the evaluation of $\mathrm{RaC}$ for the aggregate risk of portfolios. Let $X=\left(X_{1}, \ldots, X_{n}\right)$ be a portfolio of multivariate risks, where the marginal risks $X_{i}$ have distributions $F_{i}(x), i=1, \ldots, n$. In a first step, one is interested in the maximum $\mathrm{RaC}$ for the aggregate risk $S(X)=X_{1}+\ldots$ $+X_{n}$ whenever $\boldsymbol{X} \in D\left(F_{1}, \ldots, F_{n}\right)$, the set of all multivariate risks with given marginals $F_{i}(x)$. It will be shown below that the maximum $\mathrm{RaC}$ is attained when the margins $X_{i}$ show the strongest possible dependence structure, an extremal situation for which one says that $X_{1}, \ldots, X_{n}$ are mutually comonotonic.

A multivariate loss $\left(X_{1}, \ldots, X_{n}\right)$ is called comonotonic whenever an increase of a single loss $X_{i}\left(\omega_{1}\right)<X_{i}\left(\omega_{2}\right)$ for two events $\omega_{1}, \omega_{2}$ implies a nondecrease of all other losses $X_{j}\left(\omega_{1}\right) \leq X_{j}\left(\omega_{2}\right), j \neq i$ (Schmeidler (1986), Yaari (1987)). For $X \in D\left(F_{1}, \ldots, F_{n}\right)$ this is exactly the case when $X=\left(F_{1}^{-1}(U), \ldots, F_{n}^{-1}(U)\right)$ with $U$ a uniform $(0,1)$ random variable noting that $F_{i}^{-1}(U)$ has distribution $F_{i}$ and $F_{i}^{-1}$ is increasing for all $i$. The distribution $F$ of a comonotonic random vector is determined by its marginal distributions $F_{i}$ through the relationship $F\left(x_{1}, \ldots, x_{n}\right)=\min _{1 \leq i \leq n}\left\{F_{i}\left(x_{i}\right)\right\}$. Mathematically, four equivalent defining conditions of comonotonicity can be given.

Definition 2.1. (Bäuerle and Müller (1998)) The components of a random vector $\boldsymbol{X}=\left(X_{1}, \ldots, X_{n}\right) \in D\left(F_{1}, \ldots, F_{n}\right)$ are called mutually comonotonic if any of the following equivalent conditions hold:

(C1) The multivariate distribution $F\left(x_{1}, \ldots, x_{n}\right)$ of $\left(X_{1}, \ldots, X_{n}\right)$ identifies with the so-called Fréchet upper bound $F\left(x_{1}, \ldots, x_{n}\right)=\min _{1 \leq i \leq n}\left\{F_{i}\left(x_{i}\right)\right\}$.

(C2) There exists a random variable $Z$ and non-decreasing real functions $u_{1}, \ldots$, $u_{n}$ such that $\left(u_{l}(Z), \ldots, u_{n}(Z)\right)$ has the distribution $F$. 
(C3) The random vector $\left(F_{1}^{-1}(U), \ldots, F_{n}^{-1}(U)\right)$, where $U$ is uniformly distributed on $[0,1]$, has distribution $F$.

(C4) There is a random vector $\boldsymbol{X}$, distributed as $F$, such that $X_{i}\left(\omega_{1}\right)<X_{i}\left(\omega_{2}\right)$ implies $X_{j}\left(\omega_{1}\right) \leq X_{j}\left(\omega_{2}\right)$ for all $j \neq i$.

We need further the notion of supermodular order.

Definition 2.2. A random vector $\mathbf{X}$ precedes $\mathbf{Y}$ in the supermodular order, written $\mathbf{X} \leq_{s m} \mathbf{Y}$, if $E[f(X)] \leq E[f(Y)]$ for all supermodular functions $f$ such that the expectations exist, where $\mathrm{f}$ is called supermodular if

$$
f(x \wedge y)+f(x \vee y) \geq f(x)+f(y) \text { for all } x, y, \in R^{n},
$$

with the notation $\left(x_{1}, \ldots, x_{n}\right) \wedge\left(y_{1}, \ldots, y_{n}\right)=\left(x_{1} \wedge y_{1}, \ldots, x_{n} \wedge y_{n}\right), \wedge$ the minimum operator, and $\left(x_{1}, \ldots, x_{n}\right) \vee\left(y_{1}, \ldots, y_{n}\right)=\left(x_{1} \vee y_{1}, \ldots, x_{n} \vee y_{n}\right), \vee$ the maximum operator.

Intuitively the notion of supermodular function can be grasped as follows. Let $x_{1}, \ldots, x_{n}$ be $n$ individual losses in a portfolio, and let $f\left(x_{1}, \ldots, x_{n}\right)$ be the aggregate loss caused by these losses. Then supermodularity of the function $f$ means that the influence on the aggregate loss of an increase of a single loss is greater, the higher the other losses are. In the literature supermodular functions are also called superadditive, and have been originally studied in applied mathematics and operations research (e.g. Marshall and Olkin (1979)). They have been extensively applied in economics (e.g. Topkis (1998)). The related supermodular order allows for a comparison of the strength of dependence between random vectors. Its origin in the statistical literature can be traced back to Block and Sampson (1988), Joe (1990), Meester and Shanthikumar (1993), Szekli et al. (1994), Shaked and Shanthikumar (1997). Actuarial applications of this order are discussed in Müller (1997), Bäuerle and Müller (1998), Goovaerts and Dhaene (1999).

To compare the riskiness of portfolios, one says that a portfolio $X=\left(X_{1}, \ldots\right.$, $\left.X_{n}\right)$ is less risky than a portfolio $Y=\left(Y_{1}, \ldots, Y_{n}\right)$ if the corresponding aggregate risks $S(X)=X_{1}+\ldots+X_{n}$ and $S(Y)=Y_{1}+\ldots+Y_{n}$ are stop-loss ordered, that is $S(X) \leq_{\mathrm{sl}} S(Y)$. A sufficient condition for this is the supermodular order.

Theorem 2.1. Let $\boldsymbol{X}=\left(X_{1}, \ldots, X_{n}\right)$ and $\boldsymbol{Y}=\left(Y_{1}, \ldots, Y_{n}\right)$ be random vectors in $D\left(F_{1}\right.$, $\left.\ldots, F_{n}\right)$ such that $\mathbf{X} \leq_{\mathrm{sm}} \mathbf{Y}$, then one has $S(X) \leq_{\mathrm{sl}} S(Y)$.

Proof. This is shown in Müller (1997), Theorem 3.1. $\diamond$

The significance of the supermodular order for economic risk capital calculations is now immediate. Given two portfolios $X, Y \in D\left(F_{1}, \ldots, F_{n}\right)$ such that $\mathbf{X} \leq_{\mathrm{sm}} \mathbf{Y}$, it is possible to compare the $\mathrm{RaC}$ of the aggregate risk $S(X)$ with the $\mathrm{RaC}$ of the aggregate risk $S(Y)$.

Corollary 2.1. Let $\boldsymbol{X}=\left(X_{1}, \ldots, X_{n}\right)$ and $\boldsymbol{Y}=\left(Y_{1}, \ldots, Y_{n}\right)$ be random vectors in $D\left(F_{1}\right.$, $\ldots, F_{n}$ ) such that $\mathbf{X} \leq_{\mathrm{sm}} \mathbf{Y}$, then one has $\operatorname{Ra} C_{a}[S(X)] \leq \operatorname{Ra} C_{a}[S(Y)]$ for all $a \in[0,1]$. 
Proof. This is an immediate consequence of Theorem 2.1 and Theorem 1.1. $\diamond$ Even more, one obtains that the portfolio $X^{c}=\left(F_{1}^{-1}(U), \ldots, F_{1}^{-1}(U)\right) \in D\left(F_{1}, \ldots, F_{n}\right)$ with mutually comonotonic margins yields the maximum RaC.

Theorem 2.2. The maximum $\mathrm{RaC}$ for the aggregate risk of a portfolio with fixed marginal risks is attained at the portfolio with mutually comonotonic components, that is one has

$$
\max _{X \in D\left(F_{1}, \ldots, F_{n}\right)}\left\{R a C_{a}[S(X)]\right\}=\operatorname{Ra} C_{a}\left[S\left(X^{c}\right)\right] .
$$

Proof. By the inequality of Lorentz (1953) (e.g. Theorem 5 in Tchen (1980)), one knows that $\mathbf{X} \leq_{\mathrm{sm}} \mathbf{X}^{\mathbf{c}}$ for all $\boldsymbol{X} \in D\left(F_{1}, \ldots, F_{n}\right)$. The result follows by Corollary 2.1. Alternatively, it is possible to prove directly that $\mathbf{X} \leq_{\mathrm{sl}} \mathbf{X}^{\mathbf{c}}$ for all $\boldsymbol{X} \in$ $D\left(F_{1}, \ldots, F_{n}\right)$ as shown by Goovaerts et al. (2000) (see also Dhaene et al. (2000), Corollary 6). Then Theorem 1.1 implies the result. $\diamond$

This result means that comonotonicity, which displays the strongest possible dependence structure, corresponds to the riskiest portfolio under all portfolios with the same marginal risks and requires the maximum $\mathrm{RaC}$ under all these portfolios. It is further remarkable that under a simple regularity condition the maximum $\mathrm{RaC}$ is an additive functional.

Theorem 2.3. Let $\boldsymbol{X}^{c}=\left(X_{1}, \ldots, X_{n}\right)$ be a portfolio of mutually comonotonic risks with absolutely continuous marginal distributions $F_{i}(x), i=1, \ldots, n$. Then the $\mathrm{RaC}$ functional satisfies the additive property

$$
\operatorname{RaC}_{a}\left[S\left(X^{c}\right)\right]=\sum_{i=1}^{n} \operatorname{RaC}_{a}\left[X_{i}\right]
$$

Proof. Denote by $F_{s}(x)$ the distribution of $S\left(X^{c}\right)$. Consider the quantiles $d=$ $Q_{S}(\alpha), d_{i}=Q_{x_{i}}(\alpha), i=1, \ldots, n$, and the stop-loss transforms $\pi_{s}(x), \pi_{i}(x):=\pi_{x_{i}}(x)$, $i=1, \ldots, n$. By the comonotonic assumption, the quantiles and stop-loss transforms behave additively, that is one has $d=\sum_{i=1}^{n} d_{i}$ (e.g. Landsberger and Meilijson (1994), Denneberg (1994), Kaas et al. (2000)) and $\pi_{s}(d)=\sum_{i=1}^{n} \pi_{i}\left(d_{i}\right)$ (Dhaene et al. (2000), Theorem 8 , special case of absolutely continuous distributions, or Kaas et al. (2000)). The assertion follows from (1.3) using the relationship $\left(1-F_{X}(x)\right) \cdot m_{X}(x)=\pi_{X}(x)$ between the mean excess function and the stop-loss transform by means of the equalities

$$
\operatorname{RaC}_{a}\left[S\left(X^{c}\right)\right]=d+\frac{1}{\varepsilon} \cdot \pi_{s}(d)=\sum_{i=1}^{n}\left\{d_{i}+\frac{1}{\varepsilon} \cdot \pi_{i}\left(d_{i}\right)\right\}=\sum_{i=1}^{n} \operatorname{Ra} C_{a}\left[X_{i}\right] . \diamond .
$$

\section{Remark 2.1.}

As pointed out by a referee, the additive relation (2.3) is a special case of a more general result due to Dellacherie (1970) and quoted in Schmeidler 
(1986). Let $A$ be a $\sigma$-algebra of subsets of a set $S$, and $\chi$ the set of all bounded real-valued $\boldsymbol{A}$-measurable functions on $S$. For a monotone set function $v$ on $S$ such that $v(\varnothing)=0, v(S)=1$, and a non-negative real valued function $X \in \chi$, consider the Choquet integral $H_{v}[X]=\int_{s} X d v=\int_{0}^{\infty} v(X>x) d x$. Dellacherie's result states that if $X, Y \in \chi$ are comonotonic, then $H_{v}[X+Y]=H_{v}[X]+H_{v}[Y]$. In the special case of a probability space $(\Omega, P, A)$, consider the distortion function $g_{a}(x)=\min \left\{\frac{x}{1-a}, 1\right\}$ and the set function $v=g_{a}{ }^{\circ} P$. With (1.6) one obtains $\operatorname{RaC}_{a}[X]=\int_{0}^{\infty} g_{a}[P(X>x)] d x=H_{v}[X]$. The additivity (2.3) for comonotonic risks follows from Dellacherie's result. However, note that our Theorem 2.3 is not restricted to bounded random variables, an essential assumption in Schmeidler's paper.

An interesting problem concerns the impact of various "positive" dependence structures between risks $X_{1}, \ldots, X_{n}$ on the evaluation of $\mathrm{RaC}$ for the aggregate risk $S(X)=X_{1}+\ldots+X_{n}$. Independent risks with an aggregate denoted by $S^{i}=X_{1} \oplus \ldots \oplus X_{n}$ and comonotonic risks with an aggregate $S^{c}=X_{1}+{ }_{c} \ldots+{ }_{c} X_{n}$ are two extreme cases of primary importance. Let us motivate this assertion. In virtue of Corollary 2.1 and Theorem 2.2 it seems reasonable to restrict the attention to positive dependent portfolios $X \in D\left(F_{1}, \ldots, F_{n}\right)$ satisfying the supermodular inequality $\mathbf{X}^{\mathbf{i}} \leq_{\mathrm{sm}} X \leq_{\mathrm{sm}} \mathbf{X}^{\mathbf{c}}$, which implies $S^{i} \leq_{s l} S(X) \leq_{s l} S^{c}$ and $S^{i} \leq_{R a C} S(X) \leq_{R a C} S^{c}$. As an example, the family of multivariate elliptically contoured distributions is increasing in the supermodular order as the correlation increases (Block and Sampson (1988)). Portfolios satisfying only the stop-loss inequality $S^{i} \leq_{s l} S(X) \leq_{s l} S^{c}$, which by Theorem 1.1 is sufficient to imply $S^{i} \leq_{R a C} S(X) \leq_{R a C} S^{c}$, might also be of interest (e.g Bäuerle and Müller (1998), Section 4).

It is well-known that the stop-loss order relation $S^{i} \leq_{s l} S^{c}$ implies a considerable difference between the corresponding stop-loss premiums. However, the quantitative impact of this relation on the evaluation of $\mathrm{RaC}$ has not yet been examined. The additive property of Theorem 2.3 is of evident help for the quantitative analysis of the property $R a C_{a}\left[S^{i}\right] \leq R a C_{a}\left[S^{c}\right]$. Since insurance risks are often quite well approximated by gamma distributed risks or translations thereof (e.g. Seal (1977), Dufresne et al. (1991), Dickson and Waters (1993)), we will restrict ourselves in the present paper to a quantitative evaluation of this inequality for gamma risks. Since the exact distribution of sums of independent gamma random variables is not very well-known among actuaries, the next Section is of additional independent interest.

\section{SUMS OF INDEPENDENT GAMMA RANDOM VARIABLES}

Gamma distributions, which include the exponential, Erlang and chi-square distributions, are among the most important distributions widely used in applications. They are also of great importance in theoretical work. Thorin 
(1977) introduced the class of generalized gamma convolutions, defined as the smallest class of distributions on the positive real line that contains the gamma distributions and is closed with respect to convolution and weak limits, to prove the infinite divisibility of many distributions. The class of generalized gamma convolutions is surprisingly rich and has a remarkable structure. It has been extensively studied in the last century by Bondesson (1992).

Though not noticed in actuarial science (e.g. one misses them in Panjer and Willmot (1992)), expressions for the exact probability density of sums of independent gamma random variables are known from the statistical literature. For example, Johnson et al. (1994), pp. 384-85, refers to Mathai (1982), Moschopoulos (1985) and Sim (1992). One can add Provost (1989), which determines the exact density applying the inverse Mellin transform. The result by Sim (1992) uses the following direct elementary approach. Let $X_{i} \sim \Gamma\left(a_{i}, \beta_{i}\right), i=1, \ldots, n$, be $n$ independent gamma random variables with densities

$$
f_{i}(x)=g\left(\beta_{i} x ; \alpha_{i}\right):=\frac{\left(\beta_{i} x\right)^{a_{i}}}{\Gamma\left(\alpha_{i}\right)} \cdot \frac{e^{-\beta_{i} x}}{x}, x>0, \alpha_{i}, \beta_{i}>0 .
$$

The special case of identical scale parameters being well-known, one assumes that $\beta_{1}>\beta_{2}>\ldots>\beta_{n}$. The density of the independent sum $S_{n}=X_{1} \oplus \ldots \oplus X_{n}$ can be obtained from the convolution formula

$$
f_{s_{n}}(s)=\int_{0}^{s} f_{s_{n-1}}(t) \cdot f_{n}(s-t) d t
$$

applying mathematical induction. A calculation yields the result by Sim (1992) (see also Johnson et al. (1994), formula (17.110)):

$$
f_{s_{n}}(s)=\frac{1}{\Gamma\left(a^{(n)}\right)} \cdot\left[\prod_{i=1}^{n} \beta_{i}^{a_{i}}\right] \cdot s^{a^{(n)}-1} \cdot e^{-\beta_{1} s} \cdot \sum_{k=0}^{\infty} C_{k}^{n} \cdot \frac{\left(\alpha^{(n-1)}\right)_{k}}{k ! \cdot\left(a^{(n)}\right)_{k}} \cdot\left[\left(\beta_{1}-\beta_{2}\right) s\right]^{k}
$$

where $a^{(k)}=a_{1}+\ldots+a_{k},(c)_{k}=\frac{\Gamma(c+k)}{\Gamma(c)}$, and

$$
C_{k}^{i}=\left\{\begin{array}{l}
1, i=2 \\
\sum_{j=0}^{k} C_{j}^{i-1} \cdot\left[\begin{array}{l}
k \\
j
\end{array}\right] \cdot \frac{\left(a^{(i-2)}\right)_{j}}{\left(a^{(i-1)}\right)_{j}} \cdot\left[\frac{\beta_{n-i+2}-\beta_{n-i+3}}{\beta_{n-i+1}-\beta_{n-i+2}}\right]^{j}, i=3, \ldots, n .
\end{array}\right.
$$

A rearrangement shows that (3.3) is an infinite linear combination of gamma densities with the same scale parameter $\beta_{1}$, a property already observed by Provost (1989). Applying another elementary approach, we obtain below a new similar representation of the exact probability density, which differs from the results by Provost (1989) and Sim (1992). 
Theorem 3.1. Let $X_{i} \sim \Gamma\left(a_{i}, \beta_{i}\right)$ be $n$ independent gamma random variables such that $\beta_{1}>\beta_{2}>\ldots>\beta_{n}$. Then the density of the independent sum $S_{n}=X_{1} \oplus$ $\ldots \oplus X_{n}$ is analytically described by the infinite series

$$
\begin{aligned}
& f_{s_{n}}(s)=A_{n} \cdot \sum_{k=0}^{\infty} C_{k}^{n} \cdot g\left(\beta_{1} s ; a^{(n)}+k\right), \text { with } \\
& a^{(n)}=a_{1}+\ldots+a_{n}, A_{n}=\prod_{j=2}^{n}\left[\frac{\beta_{j}}{\beta_{1}}\right]^{a_{j}}, n \geq 2, A_{1}=1, \\
& C_{k}^{i}=\left\{\begin{array}{l}
\left(1-\frac{\beta_{2}}{\beta_{1}}\right]^{k} \cdot \frac{\left(a_{2}\right)_{k}}{k !}, i=2 \\
\sum_{j=0}^{k} C_{j}^{i-1} \cdot\left(1-\frac{\beta_{i}}{\beta_{1}}\right]^{k-j} \cdot \frac{\left(a_{i}\right)_{k-j}}{(k-j) !}, i=3, \ldots, n
\end{array}\right. \\
& C_{k}^{1}=0 \quad k=1,2, \ldots, \quad C_{0}^{i}=1 \quad i=1, \ldots, n .
\end{aligned}
$$

Proof. This is shown through induction. Clearly, the series representation holds for $n=1$. By induction, assume the representation holds for the index $n$ and show it for the index $n+1$. For convenience set

$$
S_{n}=X_{n} \oplus S_{n-1} \in(0, \infty), R_{n}=\frac{X_{n}}{S_{n}} \in(0,1) .
$$

Applying the standard method of transformation of random variables based on Jacobians (e.g. Fisz (1973), p. 77), the density of the sum $S_{n}$ is determined recursively by the formulas

$$
\begin{aligned}
& f_{s_{n}}(s)=\int_{0}^{1} f_{\left(s_{n}, R_{n}\right)}(s, r) d r \\
& f_{\left(s_{n}, R_{n}\right)}(s, r)=s \cdot f_{X_{n}}(r s) \cdot f_{s_{n-1}}((1-r) s) .
\end{aligned}
$$

Using this and the induction assumption, one obtains

$$
f_{s_{n+1}}(s)=A_{n} \cdot \sum_{k=0}^{\infty} C_{k}^{n} \cdot s^{a^{(n+1)}+k-1} \cdot e^{-\beta_{1} s} \cdot \frac{\beta_{1}^{a^{(n)}+k}}{\Gamma\left(a^{(n)}+k\right)} \cdot I(n, k),
$$

with

$$
\begin{aligned}
& I(n, k)=\int_{0}^{1} r^{a_{n+1}-1} \cdot(1-r)^{\alpha^{(n)}+k-1} \cdot e^{-\left(\beta_{n+1}-\beta_{1}\right)^{r s}} d r \\
& =\sum_{j=0}^{\infty} \frac{\left(\beta_{1}-\beta_{n+1}\right)^{j}}{j !} \cdot s^{j} \cdot \frac{\Gamma\left(\alpha^{(n)}+k\right) \Gamma\left(\alpha_{n+1}+j\right)}{\Gamma\left(\alpha^{(n+1)}+k+j\right)} .
\end{aligned}
$$


Through rearrangement it follows that

$$
\begin{aligned}
& f_{s_{n+1}}(s)=A_{n} \cdot\left[\frac{\beta_{n+1}}{\beta_{1}}\right]^{a_{n+1}} \cdot \sum_{k=0}^{\infty} C_{k}^{n} \cdot\left\{\sum_{j=0}^{\infty} \frac{\left(1-\frac{\beta_{n+1}}{\beta_{1}}\right)^{j} \Gamma\left(a_{n+1}+j\right)}{j ! \Gamma\left(a_{n+1}\right)} \cdot g\left(\beta_{1} s ; a^{(n+1)}+k+j\right)\right\} \\
& =A_{n+1} \cdot \sum_{k=0}^{\infty} C_{k}^{n+1} \cdot g\left(\beta_{1} s ; a^{(n+1)}+k\right) .
\end{aligned}
$$

The analytical formula (3.5) is shown. $\diamond$

\section{Remark 3.1.}

Though the coefficients of $g\left(\beta_{1} s ; a^{(n)}+k\right)$ in (3.3) and (3.5) are evaluated using different expressions, they are identical. However, the formulas (3.6) are more symmetric and simpler, and for this reason they should be preferred.

Using the incomplete gamma function defined by

$$
G(\beta x ; \alpha)=\frac{1}{\Gamma(a)} \cdot \int_{0}^{\beta x} t^{\alpha-1} e^{-t} d t,
$$

the distribution function of an independent gamma sum is through integration of (3.5) equal to

$$
F_{s_{n}}(s)=A_{n} \cdot \sum_{k=0}^{\infty} C_{k}^{n} \cdot G\left(\beta_{1} s ; a^{(n)}+k\right) .
$$

The evaluation of $\mathrm{RaC}$ for portfolios of independent gamma risks requires an analytical expression for the stop-loss transform of $S_{n}$.

Corollary 3.1. The stop-loss transform $\pi_{s_{n}}(d)=E\left[\left(S_{n}-d\right)_{+}\right]$of a sum $S_{n}=X_{1} \oplus$ $\ldots \oplus X_{n}$ of $n$ independent gamma random variables $X_{i} \sim \Gamma\left(a_{i}, \beta_{i}\right), i=1, \ldots, n$, such that $\beta_{1}>\beta_{2}>\ldots>\beta_{n}$, is determined by the analytical formula

$$
\pi_{s_{n}}(d)=E\left[S_{n}\right]-d \cdot \bar{F}_{s_{n}}(d)-\frac{1}{\beta_{1}} \cdot A_{n} \cdot \sum_{k=0}^{\infty}\left(a^{(n)}+k\right) \cdot C_{k}^{n} \cdot G\left(\beta_{1} d ; a^{(n)}+k+1\right)
$$

Proof. This follows without difficulty noting that $\pi_{s_{n}}(d)=E\left[S_{n}\right]-d \cdot \bar{F}_{s_{n}}(d)-$ $\int_{0}^{d} s f_{s_{n}}(s) d s$, and $\int_{0}^{d} s g(\beta s ; a) d s=\frac{\alpha}{\beta} G(\beta d ; a+1) . \diamond$ 


\section{Remark 3.2.}

The special case $\beta_{1}=\ldots=\beta_{\mathrm{n}}=\beta$ of identical scale parameters is well-known. In this situation, the above formulas are replaced by the very simple ones

$$
\begin{aligned}
& f_{s_{n}}(s)=g\left(\beta s ; a^{(n)}\right), F_{s_{n}}(s)=G\left(\beta s ; a^{(n)}\right), \\
& \pi_{s_{n}}(d)=\left(\frac{a^{(n)}}{\beta}-d\right) \cdot \bar{F}_{s_{n}}(d)+\frac{d}{\beta} \cdot f_{s_{n}}(s),
\end{aligned}
$$

where the last one is obtained through partial integration.

\section{NUMERICAL EXAMPLES}

First, let us calculate $\mathrm{RaC}$ for portfolios of independent gamma risks. Given the loss tolerance level $\varepsilon$, first determine using (3.13) the solution $d_{\varepsilon}$ of the equation $F_{S_{n}}\left(d_{\varepsilon}\right)=1-\varepsilon$. Inserting the obtained value in (3.14), one obtains the formula

$$
\begin{aligned}
& \operatorname{RaC}_{1-\varepsilon}\left[S_{n}^{i}\right]=d_{\varepsilon}+\frac{1}{\varepsilon} \cdot \pi_{s_{n}}\left(d_{\varepsilon}\right) \\
& =\frac{1}{\varepsilon} \cdot\left[E\left[S_{n}\right]-\frac{1}{\beta_{1}} \cdot A_{n} \cdot \sum_{k=0}^{\infty}\left(a^{(n)}+k\right) \cdot C_{k}^{n} \cdot G\left(\beta_{1} d_{\varepsilon} ; \alpha^{(n)}+k+1\right)\right] .
\end{aligned}
$$

In the special case $\beta_{1}=\ldots=\beta_{\mathrm{n}}=\beta$ of Remark 3.2 , the quantile $d_{\varepsilon}$ is solution of $G\left(\beta d_{\varepsilon} ; \alpha^{(n)}\right)=1-\varepsilon$ and (4.1) simplifies to

$$
R a C_{1-\varepsilon}\left[S_{n}^{i}\right]=E\left[S_{n}\right] \cdot\left\{1+\frac{1}{\varepsilon} \cdot \frac{d_{\varepsilon}}{a^{(n)}} \cdot g\left(\beta d_{\varepsilon} ; \alpha^{(n)}\right)\right\} .
$$

Second, let us calculate $\mathrm{RaC}$ for portfolios of comonotonic gamma risks. The evaluation uses the additive property of Theorem 2.2. For $i=1, \ldots, n$, determine the solution $d_{i, \varepsilon}$ of $G\left(\beta_{i} d_{i, \varepsilon} ; \alpha_{i}\right)=1-\varepsilon$. Replacing $\alpha^{(n)}$ by $\alpha_{i}$ in (3.15) one gets

$$
\pi_{i}\left(d_{i, \varepsilon}\right)=\varepsilon \cdot\left(E\left[X_{i}\right]-d_{i, \varepsilon}\right)+\frac{d_{i, \varepsilon}}{\beta_{i}} \cdot g\left(\beta_{i} d_{i, \varepsilon} ; a_{i}\right) .
$$

It follows that

$$
\begin{aligned}
& \operatorname{RaC}_{1-\varepsilon}\left[S_{n}^{c}\right]=\sum_{i=1}^{n} \operatorname{Ra} C_{1-\varepsilon}\left[X_{i}\right]=\sum_{i=1}^{n}\left\{d_{i, \varepsilon}+\frac{1}{\varepsilon} \cdot \pi_{i}\left(d_{i, \varepsilon}\right)\right\} \\
& =E\left[S_{n}\right]+\frac{1}{\varepsilon} \cdot \sum_{i=1}^{n} \frac{d_{i, \varepsilon}}{\beta_{i}} \cdot g\left(\beta_{i} d_{i, \varepsilon} ; a_{i}\right) .
\end{aligned}
$$


Example 4.1: independence versus comonotonic assumption

In the special case $\beta_{1}=\ldots=\beta_{n}=\beta$ of Remark 3.2, a comparison of (4.2) and (4.3) yields the difference formula for $\mathrm{RaC}$ :

$$
\beta \varepsilon \cdot\left(R a C_{1-\varepsilon}\left[S_{n}^{c}\right]-R a C_{1-\varepsilon}\left[S_{n}^{i}\right]\right)=\sum_{i=1}^{n} \frac{d_{i, \varepsilon}}{\beta_{i}} \cdot g\left(\beta_{i} d_{i, \varepsilon} ; a_{i}\right)-d_{\varepsilon} \cdot g\left(\beta d_{\varepsilon} ; a^{(n)}\right)
$$

where $G\left(\beta d_{\varepsilon} ; a^{(n)}\right)=1-\varepsilon$ and $G\left(\beta_{i} d_{i, \varepsilon} ; \alpha_{i}\right)=1-\varepsilon, i=1, \ldots, n$. A numerical illustration for the exponential case $\beta=1, \alpha_{1}=\ldots=\alpha_{n}=1$, is summarized in Table 4.1 below. In this situation $\operatorname{RaC}_{1-\varepsilon}\left[S_{n}^{c}\right]=n \cdot\{1-\ln (\varepsilon)\}$ depends linearly on the number of exponential risks. The difference increases non-linearly according to the formula

$$
R a C_{1-\varepsilon}\left[S_{n}^{c}\right]-R a C_{1-\varepsilon}\left[S_{n}^{i}\right]=\frac{1}{\varepsilon} \cdot\left\{n \cdot \varepsilon \cdot[-\ln (\varepsilon)]-\frac{d_{\varepsilon}^{n} e^{-d_{r}}}{(n-1) !}\right\},
$$

where $G\left(d_{\varepsilon} ; n\right)=1-\varepsilon$. As an interesting observation, one notes for increasing $n$ a decreasing percentage increase of $\operatorname{RaC}_{1-\varepsilon}\left[S_{n}^{i}\right]$ over the $\varepsilon$-range between 0.05 and 0.001 .

TABLE 4.1

RAC FOR EXPONENTIAL RISK PORTFOLIOS UNDER INDEPENDENCE (I) AND COMONOTONIC (C) ASSUMPTION

\begin{tabular}{lrrrrrr}
\hline \hline & \multicolumn{2}{c}{$\boldsymbol{\varepsilon}=\mathbf{0 . 0 5}$} & \multicolumn{2}{c}{$\boldsymbol{\varepsilon}=\mathbf{0 . 0 1}$} & \multicolumn{2}{c}{$\boldsymbol{\varepsilon}=\mathbf{0 . 0 0 1}$} \\
\cline { 2 - 7 } & \multicolumn{1}{c}{ (i) } & (c) & \multicolumn{1}{c}{ (i) } & \multicolumn{1}{c}{ (c) } & \multicolumn{1}{c}{ (i) } & (c) \\
\hline 1 & 4 & 4 & 5.6 & 5.6 & 7.9 & 7.9 \\
2 & 5.9 & 8 & 7.8 & 11.2 & 10.3 & 15.8 \\
3 & 7.6 & 12 & 9.6 & 16.8 & 12.4 & 23.7 \\
4 & 9.2 & 16 & 11.4 & 22.4 & 14.3 & 31.6 \\
5 & 10.7 & 20 & 13 & 28 & 16.1 & 39.5 \\
10 & 17.6 & 40 & 20.5 & 56.1 & 24.2 & 79.1 \\
20 & 30.3 & 79.9 & 34 & 112.1 & 38.6 & 158.2 \\
50 & 65.7 & 199.8 & 70.9 & 280.3 & 77.3 & 395.4 \\
100 & 121.7 & 399.6 & 128.7 & 560.5 & 137.2 & 790.8 \\
\hline \hline
\end{tabular}

Example 4.2: sums of independent gamma risks versus gamma and normal approximations

Suppose an insurer desires to calculate $\mathrm{VaR}$ and $\mathrm{RaC}$ for a portfolio of $n$ independent risks, which follow a classical risk model. Each risk $X_{i}=Y_{i, 1}+\ldots$ $+Y_{i, N_{i}}$ has a compound Poisson distribution, where $N_{i}$ is Poisson distributed 
and the $Y_{i, j}$ 's are the individual claims. Assume that one knows the expected number of claims $\lambda_{i}=E\left[N_{i}\right]$, as well as the first and second moments $v_{i}=E\left[Y_{i, 1}\right]$ $m_{i, 2}=E\left[Y_{i, 1}^{2}\right]$ of the severity distributions, $i=1, \ldots, n$. Let $k_{i}=c_{i} \cdot \lambda_{i}^{-\frac{1}{2}}$, with $c_{i}=$ $\sqrt{m_{2, i}} \cdot v_{i}^{-1}$, be the coefficient of variation of $X_{i}$. As mentioned previously, it is often possible to assume that $X_{i}$ is gamma distributed with parameters

$$
\alpha_{i}=\frac{1}{k_{i}^{2}}=\frac{\lambda_{i}}{c_{i}^{2}}, \quad \beta_{i}=\frac{1}{c_{i}^{2}} \cdot \frac{1}{v_{i}}, i=1, \ldots, n .
$$

The risk $S_{n}=X_{1} \oplus \ldots \oplus X_{n}$ of the portfolio is again compound Poisson distributed with corresponding parameters

$$
\lambda=\sum_{i=1}^{n} \lambda_{i}, \quad v=\sum_{i=1}^{n}\left[\frac{\lambda_{i}}{\lambda}\right] \cdot v_{i}, \quad k=c \cdot \lambda^{-\frac{1}{2}}, c^{2}=\sum_{i=1}^{n}\left[\frac{\lambda_{i}}{\lambda}\right] \cdot\left[\frac{v_{i}}{v}\right]^{2} \cdot c_{i}^{2} .
$$

Now, it is possible to approximate $S_{n}$ either by a sum of $n$ independent gamma risks with the parameters $\alpha_{i}, \beta_{i}$ in (4.6) or by a gamma risk with parameters $a=\frac{\lambda}{c^{2}}, \beta=\frac{1}{c^{2}} \cdot \frac{1}{v}$ as defined in (4.7). To illustrate, we compare the VaR and $\mathrm{RaC}$ values of these approximations with the values obtained from a normal approximation for portfolios of 5 risks with parameters (typical for the aggregate claims of collectives of life insurance policies):

$$
\begin{aligned}
& \left(\lambda_{1}, \ldots, \lambda_{5}\right)=m \cdot(1,1,1,1,1), m=1,2,5,10,20,50, \\
& \left(v_{1}, \ldots, v_{5}\right)=(2,2,1,3,2),\left(c_{i}, \ldots, c_{5}\right)=(1.25,1.75,2.5,1.5,2) .
\end{aligned}
$$

The parameters for the overall gamma approximation are by (4.7) equal to $\lambda=$ $5 m, v=2, c=1.74642$. Table 4.2 shows that the VaR and $\mathrm{RaC}$ values of both gamma approximations differ only slightly, but the normal approximation underestimates systematically these values, especially for small $\lambda_{i}$ 's and more considerably for $\mathrm{RaC}$ than for VaR.

TABLE 4.2

VAR AND RAC COMPARISONS BY FIXED $\varepsilon=0.05$

\begin{tabular}{lcccccc}
\hline \hline \multirow{3}{*}{$\mathbf{m}$} & \multicolumn{2}{c}{ VaR } & & \multicolumn{2}{c}{ RaC } \\
\cline { 2 - 7 } & $\begin{array}{r}\text { normal } \\
\text { approx. }\end{array}$ & $\begin{array}{r}\text { gamma } \\
\text { approx. }\end{array}$ & $\begin{array}{c}\text { sum of ind. } \\
\text { gamma }\end{array}$ & $\begin{array}{c}\text { normal } \\
\text { approx. }\end{array}$ & $\begin{array}{r}\text { gamma } \\
\text { approx. }\end{array}$ & $\begin{array}{c}\text { sum of ind, } \\
\text { gamma }\end{array}$ \\
\hline 1 & 22.8 & 25.3 & 25.3 & 26.1 & 32.1 & 32.4 \\
2 & 38.2 & 40.9 & 41.0 & 42.8 & 49.1 & 49.5 \\
5 & 78.7 & 81.8 & 81.9 & 86.0 & 92.6 & 93.0 \\
10 & 140.6 & 143.8 & 144.0 & 150.9 & 157.6 & 158.1 \\
20 & 257.5 & 260.7 & 260.9 & 272.0 & 278.8 & 279.3 \\
50 & 590.8 & 594.2 & 594.4 & 613.9 & 620.7 & 621.2 \\
\hline \hline
\end{tabular}




\section{REFERENCES}

Artzner, P. (1999) Application of coherent risk measures to capital requirements in insurance. North American Actuarial Journal 3(2), 11-25.

Artzner, P., Delbaen, F., Eber, J.-M. and Heath D. (1997a) Definition of coherent measures of risk. Risk Management Symposium of the European Finance Association Meeting, Vienna, August 27-30, 1997.

Artzner, P., Delbaen, F., Eber, J.-M. and Heath D. (1997b) Thinking coherently. RISK 10, no. 11 (November 1997), 68-70.

Ballmann, M., and HüRlimanN W. (2000) Zur Modellierung des ökonomischen Risikokapitals in der Lebensversicherung. Manuscript.

BÄUERLE, N., and MüLLER A. (1998) Modeling and comparing dependencies in multivariate risk portfolios. ASTIN Bulletin 28(1), 59-76.

Blackwell, D. and Dubins L.E. (1963) A converse to the dominated convergence theorem. Illinois Journal of Mathematics 7, 508-514.

BLoCK, H.W. and SAMPSON A.R. (1988) Conditionally ordered distributions. Journal of Multivariate Analysis 27, 91-104.

BONDESSON, L. (1992) Generalized gamma convolutions and related classes of distributions. Lecture Notes in Statistics 76. Springer-Verlag.

Dellacherie, C. (1970) Quelques commentaires sur les prolongements de capacités. Séminaire de Probabilités V, Strasbourg. Lecture Notes in Mathematics 191. Springer-Verlag, Berlin and New York.

DenneberG, D. (1994) Non-Additive Measure and Integral. Theory and Decision Library, Series B, vol. 27. Kluwer Academic Publishers.

Denuit, M., Dhaene, J. and Van Wouve M. (1999) The Economics of Insurance: a review and some recent developments. Bulletin of the Swiss Association of Actuaries, 137-175.

Dhaene, J., Wang, S., Young, V. and Goovaerts M.J. (2000) Comonotonicity and maximal stop-loss premiums. Bulletin of the Swiss Association of Actuaries, 99-113.

DicksON, D.C.M., and WATERS H.R. (1993) Gamma processes and finite time survival probabilities. ASTIN Bulletin 23(1), 259-272.

Dubins, L.E. and Gilat D. (1978) On the distribution of the maxima of martingales. Transactions of the American Mathematical Society 68, 337-38.

Dufresne, F., Gerber, H.U. and SHIU E.S.W. (1991) Risk theory with the Gamma process ASTIN Bulletin 21(2), 177-192.

EMBrechTS, P. (1995) A survival kit to quantile estimation. UBS Quant Workshop, Zürich.

Embrechts, P., KlüPPelberG, C. and MikosCi Th. (1997) Modelling Extremal Events for Insurance and Finance. Springer, Berlin.

Embrechts, P., McNeil, A. and Straumann D. (1998) Correlation and dependency in Risk Management: properties and pitfalls. Preprint, ETHZ.

FISZ, M. (1973) Wahrscheinlichkeitrechnung und mathematische Statistik. VEB Deutscher Verlag der Wissenschaften. Berlin.

GoovaerTs, M.J. and DHAENe J. (1999) Supermodular ordering and stochastic annuities. Insurance: Mathematics and Economics 24, 281-290.

Goovaerts, M.J., Dhaene, J. and De SchePPER A. (2000) Stochastic upper bounds for present value functions. Journal of Risk and Insurance Theory 67, 1-14.

HARDY, G.H. and LiTTLEWOOD J.E. (1930) A maximal theorem with function-theoretic applications. Acta Mathematica 54, 81-116.

HÜRLIMANN, W. (1998a) Risk measurement and financial gain: a paradox and a reconciliation. Proceedings $2^{\text {nd }}$ International Congress on Insurance: Mathematics and Economics, Lausanne, July 20-22, 1998. Unpublished improved manuscript.

HÜRLIMANN, W. (1998b) On distribution-free safe layer-additive pricing. Insurance: Mathematics and Economics 22, 277-85.

HÜRLIMANN, W. (1998c) On stop-loss order and the distortion pricing principle. ASTIN Bulletin 28(1), 119-34. 
HüRLIMANN, W. (1998d) Inequalities for lookback option strategies and exchange risk modelling. Proceedings of the 1st Euro-Japanese Workshop on Stochastic Modelling for Finance, Insurance, Production and Reliability, Brussels, 1998.

JoE, H. (1990) Multivariate concordance. Journal of Multivariate Analysis 35, 12-30.

JoHnson, N.L., Kotz, S. and BALAKRISHNAN N. (1994) Continuous Univariate Distributions (2nd ed.), vol. 1. J. Wiley, New York.

KaAs, R., Dhaene, J. and GoovaerTs M.J. (2000) Upper and lower bounds for sums of random variables. Paper presented at the 4th International Congress Insurance: Mathematics and Economics. Barcelona, July 24-26, 2000.

KERTZ, R.P. and RösLeR U. (1990) Martingales with given maxima and terminal distributions. Israel Journal of Mathematics 69, 173-192.

KERTZ, R.P. and RösLER U. (1992) Stochastic and convex orders and lattices of probability measures, with a martingale interpretation. Israel Journal of Mathematics 77, 129-164.

KERTZ, R.P. and RösLER U. (1993) Hyperbolic-concave functions and Hardy-Littlewood maximal functions. In: Stochastic Inequalities. IMS Lecture Notes - Monograph Series, vol. 22.

LANDSBERGer, M. and MEILIJSON I. (1994) Co-monotone allocations, Bickel-Lehman dispersion and the Arrow-Pratt measure of risk aversion. Annals Operations Research 52, 97106.

LORENTZ, G.G. (1953) An inequality for rearrangements. American Mathematical Monthly 60, 178-79.

Marshall, A.W. and OlKIN I. (1979) Inequalities, Theory of Majorization and Its Applications. Academic Press, New York.

MathaI, A.M. (1982) Storage capacity of a dam with gamma type inputs. Annals of the Institute of Statistical Mathematics 34, 591-97.

MeEster, L.E. and ShanTHIKUMAR J.G. (1993) Regularity of stochastic processes: a theory based on directional convexity. Probability in the Engineering and Informational Sciences 7 , 343-360.

MeILIJSON, I. and NȦDAs A. (1979) Convex majorization with an application to the length of critical paths. Journal of Applied Probability 16, 671-77.

Moschopoulos, P.G. (1985) The distribution of the sum of independent gamma random variables. Annals of the Institute of Statistical Mathematics 37, 541-44.

Müller, A. (1997) Stop-loss order for portfolios of dependent risks. Insurance: Mathematics and Economics 21, 219-223.

Panjer, H.H. and Willmot G.E. (1992) Insurance Risk Models. Society of Actuaries, Schaumburg, Illinois.

Provost, S.B. (1989) On sums of independent gamma random variables. Statistics 20, 583-91.

RüsChENDORF, L. (1991) On conditional stochastic ordering of distributions. Advances in Applied Probability 23, 46-63.

SCHMEIDLER, D. (1986) Integral representation without additivity. Proceedings American Mathematical Society 97, 255-261.

SCHRÖDER, M. (1996) The Value at Risk Approach - Proposals on a Generalization. Aktuarielle Ansätze für Finanz-Risiken, AFIR 1996, vol. 1, 151-170. Verlag Versicherungswirtschaft, Karlsruhe.

SEAL, H.L. (1977) Approximations to risk theory's $\mathrm{F}(\mathrm{x}, \mathrm{t})$ by means of gamma distributions. ASTIN Bulletin 9, 213-18.

Shaked, M. and Shanthikumar J.G. (1997) Supermodular stochastic orders and positive dependence of random vectors. Journal of Multivariate Analysis 61, 86-101.

SIM, C.H. (1992) Point processes with correlated gamma interarrival times. Statistics and Probability Letters 15, 135-141.

SZEKLI, R., DISNEY, R.L. and HuR S. (1994) MR/G1/1 queues with positively correlated arrival stream. Journal of Applied Probability 31, 497-514.

TCHEN, A.H. (1980) Inequalities for distributions with given marginals. The Annals of Probability 8(4), 814-27.

THORIN, O. (1977) On the infinite divisibility of the lognormal distribution. Scandinavian Actuarial Journal, 121-148. 
TOPKIS, D.M. (1998) Supermodularity and Complementarity. Frontiers of Economic Research. Princeton University Press. Princeton, New Jersey.

WANG, S. (1996) Premium calculation by transforming the layer premium density. ASTIN Bulletin 26, 71-92.

WIRCH, J.L. (1999) Raising value at risk. North American Actuarial Journal 3(2), 106-115.

YAARI, M.E. (1987) Dual theory of choice under uncertainty. Econometrica 55, 95-115.

\section{WERNER HÜRLIMANN \\ Value and Risk Management \\ Winterthur Life and Pensions \\ Postfach 300 \\ CH-8401 Winterthur \\ Switzerland}

